the production of 15 -keto- $\mathrm{PGE}_{2}$ is reduced in Cftr-deficient mice. They traced the defect to a severe reduction in the expression of 15-hydroxyprostaglandin dehydrogenase (HPGD), the enzyme that produces 15-keto$\mathrm{PGE}_{2}$ from $\mathrm{PGE}_{2}$ (ref. 1). This enzyme has not been previously suggested to be crucial to the pathophysiology of cystic fibrosis.

Together with previous studies ${ }^{4}$ demonstrating that the expression of PPAR- $\gamma$ is low in the colon, ileum and lung of Cftr-deficient mice, these findings indicate a dysfunction of the HPGD-15-keto-PGE 2 -PPAR- $\gamma$ system in cystic fibrosis. At present, we do not know how a deficiency in Cftr lowers the expression of HPGD; the next challenge in the field is to identify the mechanisms that mediate the downregulation of this enzyme and the reduced release of 15 -keto-PGE 2 in cystic fibrosis.

The present study adds another lipid to the growing list of lipids shown to be altered in Cftr-deficient cells, such as prostaglandins ${ }^{5}$, cholesterol $^{6}$ and ceramide ${ }^{7}$. In addition, Cftr requires small membrane domains enriched by sphingolipids and cholesterol, named rafts, for its physiological functions ${ }^{8}$. Determining how Cftr is involved in the metabolism of these lipids seems to be important for solving the pathophysiology of cystic fibrosis. Manipulating lipids, for instance by interfering with their production or by bypassing a defect, as in the present study using rosiglitazone, may have great clinical potential in cystic fibrosis. Therefore drugs such as rosiglitazone may be useful in studies of the treatment of cystic fibrosis.

\section{COMPETING INTERESTS STATEMENT}

The author declares no competing financial interests.

1. Harmon, G.S., Ng, D.T., Barrett, K.E., Dong, H. \& Glass, C.K. Nat. Med. 16, 313-318 (2010).

2. Perez, A. et al. Am. J. Physiol. Lung Cell. Mol. Physiol. 295, L303-L313 (2008).

3. Bajwa, P.J., Lee, J.W., Straus, D.S. \& Lystle, C. Am. J. Physiol. Gastrointest. Liver Physiol. 297, G82G89 (2009).

4. Ollero, M. et al. J. Cell. Physiol. 200, 235-244 (2004).

5. Chase, H.P. \& Dupont, J. Lancet 2, 236-238 (1978).

6. Manson, M.E., Corey, D.A., White, N.M. \& Kelley, T.J. Am. J. Physiol. Lung Cell. Mol. Physiol. 295, L809L819 (2008).

7. Teichgräber, V. et al. Nat. Med. 14, 382-391 (2008).

8. Kowalski, M.P. \& Pier, G.B. J. Immunol. 172, 418-425 (2004).

\title{
HIV vaccines: mosaic approach to virus diversity
}

\author{
Lawrence Corey \& M Juliana McElrath
}

\begin{abstract}
The extraordinary diversity of HIV is a major barrier in the path of developing a vaccine. One way forward may be mosaic antigens-biometrically designed genes that maximize overlap between sequences used in the vaccine and circulating HIV-1 strains worldwide (pages 319-323 and 324-328).
\end{abstract}

The high genetic diversity of HIV-1 presents a special challenge in HIV vaccine development. An ideal vaccine is one that can elicit host immune responses to effectively block and contain infection from the highly variable viruses circulating in the human population.

The mosaic vaccine approach offers one potential solution. This approach applies in silico algorithms to select vaccine genes or proteins that more closely match and maximize the sequences of natural HIV-1 strains worldwide. Fischer et al. ${ }^{1}$ designed such vaccines several years ago, and, in this issue of Nature Medicine, Barouch et al. ${ }^{2}$ and Santra et al. ${ }^{3}$ reveal the experimental results in rhesus macaques. The findings show that these vaccines seem to improve the diversity of immune responses to globally circulating strains of HIV-1.

Among communities in which the HIV epidemic is well established, the diversity of circulating HIV strains far exceeds the antigenic

Lawrence Corey and M. Juliana McElrath are at the Vaccine and Infectious Disease Institute, Fred Hutchinson Cancer Research Center, Seattle, Washington, USA. Lawrence Corey is in the Departments of Laboratory Medicine and Medicine and M. Juliana McElrath is in the Departments of Medicine and Global Health, University of Washington, Seattle, Washington, USA. e-mail: Icorey@u.washington.org variation of other known pathogens. Although recent findings from complete genome sequence analyses of founder transmitted viruses suggest that a single viral strain initiates infection ${ }^{4}$ (M. Rolland, S. Tovanabutra, A.C. deCamp, P.B. Gilbert, E. Sanders-Buell et al., unpublished data) more than $95 \%$ of infecting strains are unique among a diverse population of susceptible people . $^{5}$.

Almost all HIV-1 vaccines to date have included one to three natural HIV strain sequences as gene inserts in viral vectors or HIV antigens in subunit proteins. Such vaccines have been insufficient to protect against the myriad infecting strains in a population or community at risk for HIV ${ }^{6,7}$. Both neutralizing antibody and $\mathrm{T}$ cell responses after vaccination with such strains are largely focused on a few antigens specific to the immunizing strains rather than a larger number of antigens specific to the diverse circulating strains. This lack of coverage has been a major factor in the inability to achieve efficacy in either preventing infection or controlling viral replication after infection. Moreover, recent data from an HIV vaccine efficacy trial conducted in Thailand suggests that non-neutralizing antiviral antibody functions may have an unappreciated role in protection ${ }^{8}$. However, little is known about the coverage of such responses in a population infected with a diverse population of isolates.
Both human and nonhuman primate studies indicate that $\mathrm{T}$ cells contribute to the early control of viral infection. Previous findings that $\mathrm{CD}^{+} \mathrm{T}$ cells from infected people commonly recognize epitopes in conserved regions of HIV-1 Gag and Nef provided the impetus for designing immunogens to elicit $\mathrm{CD} 8^{+} \mathrm{T}$ cells that recognize a high frequency of circulating viruses through their shared conserved sequences. However, recent observations from studies of test reagents more closely matching the protein sequences of the infecting strain (as opposed to reference strains) have revealed that responses are commonly generated to the variable rather than the highly conserved regions of the proteins ${ }^{9,10}$.

Moreover, epitope mapping studies after vaccination with a replication-incompetent adenovirus serotype 5 (Ad5) vaccine containing the gag, pol and nef genes of HIV-1 indicate that, despite relatively robust immune responses, the $\mathrm{CD}^{+} \mathrm{T}$ cells elicited by vaccination commonly recognized epitopes within variable regions, even when sequences from conserved regions were included in the experimental HIV vaccine. This lack of 'matching' between vaccine-induced immunity and the antigenic phenotype of the infecting strain is probably a major explanation for the inability of this vaccine to control viral replication in vaccinated individuals.

The next generation of T cell-based vaccines will require designs with improved 
Table 1 Summary of vaccine approach and findings in the Barouch et al. ${ }^{2}$ and Santra et al. ${ }^{3}$ studies.

\begin{tabular}{|c|c|c|}
\hline Parameter & Barouch et al. ${ }^{2}$ & Santra et al. ${ }^{3}$ \\
\hline \multicolumn{3}{|l|}{ Vaccine } \\
\hline Prime and boost & Ad26 and Ad5HVR48 & DNA and vaccinia \\
\hline HIV-1 mosaic & Gag, Pol and Env & Gag, Nef \\
\hline $\begin{array}{l}\text { Valency (number of } \\
\text { synthetically derived } \\
\text { mosaics for each gene) }\end{array}$ & Bivalent & Quadrivalent \\
\hline \multicolumn{3}{|l|}{ Primary analysis } \\
\hline & $\begin{array}{l}\text { Intracellular cytokine staining } \\
\text { of CD8+ and CD4+ T cells } \\
\text { using peptide panel designed } \\
\text { to evaluate the most prevalent } \\
\text { strains of HIV-1 worldwide }\end{array}$ & $\begin{array}{l}\text { Interferon- } \gamma \text { ELISPOT of PBMCs } \\
\text { and CD } 8^{+} \text {-depleted PBMCs on } \\
\text { the basis of ten natural isolates } \\
\text { from the A, B, C, D and G } \\
\text { clades of HIV-1 }\end{array}$ \\
\hline
\end{tabular}

$\mathrm{CD}^{+} \mathrm{T}$ cell responses

\begin{tabular}{|c|c|c|}
\hline Per strain (median) & $\begin{array}{l}16 \text { (mosaic) versus } \\
5 \text { (consensus } \mathrm{M}^{\mathrm{a}} \text { ) }\end{array}$ & $\begin{array}{l}2.0 \text { (mosaic) versus } 1.0 \text { (con- } \\
\text { sensus) }\end{array}$ \\
\hline Per epitope region (median) & $\begin{array}{l}8 \text { (mosaic) versus } \\
2{\text { (consensus } M^{\mathrm{a}} \text { ) }}^{\text {(consen }}\end{array}$ & $\begin{array}{l}\text { Total and Gag greater in mosaic } \\
\text { than consensus }\end{array}$ \\
\hline $\begin{array}{l}\text { Per variants within } \\
\text { epitope region }\end{array}$ & $\begin{array}{l}\geq 2 \text { more in mosaic versus } \\
\text { consensus } \mathrm{M}^{\mathrm{a}}\end{array}$ & $\mathrm{N} / \mathrm{A}$ \\
\hline
\end{tabular}

\section{$\mathrm{CD}^{+} \mathrm{T}$ cell responses}

\begin{tabular}{|c|c|c|}
\hline Per strain (median) & $\begin{array}{l}3.7 \text { (mosaic) versus } \\
1.2 \text { (consensus) }\end{array}$ & $\begin{array}{l}4.5 \text { (mosaic) versus } 2.0 \text { (con- } \\
\text { sensus) }\end{array}$ \\
\hline Per epitope region (median) & $\begin{array}{l}3.3 \text { (mosaic) versus } \\
1.2 \text { (consensus) }\end{array}$ & $\mathrm{N} / \mathrm{A}$ \\
\hline $\begin{array}{l}\text { Per variants within } \\
\text { epitope region }\end{array}$ & & $\begin{array}{l}3 \text { (mosaic) versus } \\
1 \text { (consensus) }\end{array}$ \\
\hline
\end{tabular}

aGroup M consists of 9 distinct subtypes or clades of HIV-1: clades A, B, C, D, F, G, H, J and K. N/A, not available; ELISPOT, enzyme-linked immunospot; PBMCs, peripheral blood mononuclear cells.

The major differences between the two studies include the vaccine regimen, the analysis of immune responses and specificities, and the degree of enhanced T cell responses elicited by the mosaic vaccine as compared to those elicited by consensus or natural strain vaccines.

coverage. Such vaccines should better represent sequences circulating in the target population (frequency), induce responses that recognize multiple epitopes (breadth) and induce responses to the variants within these epitopes (depth) $)^{11}$.

Barouch et al. ${ }^{2}$ and Santra et al. ${ }^{3}$ used synthetically designed mosaic inserts encoding full-length proteins optimized to provide improved $\mathrm{T}$ cell epitope coverage. They show that, in nonhuman primates, HIV-1 vaccines based on such inserts can broaden the range of recognized epitopes and increase responses to high-frequency epitopic variants.

The two studies differed by the vaccine regimen administered, the analysis of immune responses and specificities and the degree of enhanced $\mathrm{T}$ cell responses after the mosaic vaccine in comparison with the consensus or natural strain vaccines (Table 1). Barouch et al. ${ }^{2}$ used two complementary HIV-1 mosaics containing HIV-1 gag, pol and env inserted into two separate replication-defective adenovirus vectors and performed detailed epitope mapping studies in rhesus macaques after vaccination. They showed that after one dose, the Ad26 gag-pol-env vaccine induced $\mathrm{CD}^{+}$ $\mathrm{T}$ cells and some $\mathrm{CD} 4^{+} \mathrm{T}$ cells that recognized more epitopes (breadth) as well as more variants within an epitope (depth) than Ad26 vectors expressing consensus or natural sequence antigens. A heterologous Ad5HVR48 vector boosted these responses proportionately to the prime. Additionally, binding and some neutralizing antibodies to HIV-1 envelope were elicited.

In contrast, Santra et al. ${ }^{3}$ administered a plasmid DNA prime and recombinant vaccinia virus boost with quadrivalent mosaic HIV-1 gag and nef inserts. Here, too, the synthetic mosaic antigens resulted in T cells with greater epitope breadth and variant depth among immunized macaques, albeit the response differences between the mosaic and consensus natural sequences were less apparent than those elicited by the adenovirus vector vaccines. For example, the total number of Gag- and Nefspecific epitopes recognized by $\mathrm{T}$ cells against a single strain of HIV were not statistically different (7.8 versus 5.5$)$. $\mathrm{CD} 4^{+} \mathrm{T}$ cells, which were elicited in higher frequency with the vaccinia vector than were $\mathrm{CD}^{+} \mathrm{T}$ cells, did show statistically significant greater breadth with the mosaic antigens.

Taken together, these studies provide evidence that biometrically designed synthetic genes can elicit a more diverse immune response in rhesus macaques as compared to natural or consensus strains, irrespective of the vector delivery system. They also indicate that the vector itself influences the nature of the immune response to the mosaic inserts. The differences between the two studies (Table 1) may be in part explained by the HIV-1 genes selected as inserts, the diverse major histocompatibility complex proteins of the rhesus macaques and the relatively small numbers of macaques used for the detailed analyses.

Moreover, these two studies also vividly illustrate that the reagents used to map epitopes influence the type of data obtained. The two groups did not use the same peptide reagent panels or methodologies to identify the $\mathrm{CD} 4^{+}$ and $\mathrm{CD}^{+} \mathrm{T}$ cell responses and to define the epitope specificities, and, hence, a direct comparison of the role that the vector versus inserts have in the immunological differences achieved is not possible.

These two studies provide a pathway toward eliciting antibody and $\mathrm{T}$ cell responses that would cope with the genetic diversity of HIV in a community. They also shed light on the use of biometrically designed synthetic inserts and peptide reagents to more predictably evaluate the likelihood of eliciting effective $\mathrm{CD}^{+}$and $\mathrm{CD}^{+} \mathrm{T}$ cell responses after HIV vaccination.

There are major caveats to these macaque studies in their translation to human research. To date, studies in rhesus macaques have shown greater epitope breadth after immunization than studies of similar vaccine strategies in humans. We do not know whether stochastically increasing the number of potential epitopes or the immune response to common variants in nonhuman primates will result in a more effective immune response in humans; these studies can only be defined in humans. Such studies are in the planning phase.

If follow-up studies in people of such synthetically derived inserts result in improved T cell function after vaccination, this approach could have broad implications for vaccine development. The approach has the potential to lead to improvements for vaccines against pathogens in which antigenic variation presents a major obstacle for immune-based protection, such as HIV, influenza A and hepatitis C.

COMPETING INTERESTS STATEMENT

The authors declare no competing financial interests. 
1. Fischer, W. et al. Nat. Med. 13, 100-106 (2007).

2. Barouch, D.H. et al. Nat. Med. 16, 319-323 (2010).

3. Santra, S. et al. Nat. Med. 16, 324-328 (2010).

4. Salazar-Gonzalez, J.F. et al. J. Exp. Med. 206, 12731289 (2009)
5. Celum, C. et al. Lancet 371, 2109-2119 (2008).

6. Buchbinder, S.P. et al. Lancet 372, 1881-1893 (2008).

7. Pitisuttithum, P. et al. J. Infect. Dis. 194, 1661-1671 (2006).

8. Rerks-Ngarm, S. et al. N. Engl. J. Med. 361, 2209-
2220 (2009)

9. Liu, Y. et al. J. Virol. 83, 10821-10829 (2009).

10. Goonetilleke, N. et al. J. Exp. Med. 206, 1253-1272 (2009).

11. Li, F. et al. Curr. HIV Res. 5, 97-107 (2007).

\title{
Spasticity: a switch from inhibition to excitation
}

\author{
V Reggie Edgerton \& Roland R Roy
}

\begin{abstract}
The involuntary muscle spasms that occur as a result of neuromotor disorders and spinal cord injury can have dangerous consequences for affected individuals. New findings shed light on one mechanism contributing to spasticity: limited chloride transport in motoneurons (pages 302-307).
\end{abstract}

Individuals with a spinal cord injury often suffer from involuntary contractions of muscles, or spasticity, below the site of injury. The consequences range from being relatively minor to potentially dangerous, if, for example, the contractions are severe enough to cause someone to fall from a chair. And although medications have proven to be effective in quelling symptoms of spasticity, they also can interfere with remaining motor functions. For this reason, individuals who are undergoing locomotor training as part of their rehabilitative program do not usually take antispastic drugs ${ }^{1}$. There is a great need for interventions that alleviate spasticity without limiting the potential for recovery of locomotor function.

To develop such interventions, it is crucial to understand the underlying mechanisms of spasticity. Although many processes are thought to be involved, Boulenguez et al. ${ }^{2}$ now uncover at least one mechanism that contributes to spasticity. They find that in animal models spinal cord injury is accompanied by a downregulation of the potassium-chloride co-transporter-2 (KCC2) in motoneuron membranes, which, in turn, results in a substantial positive shift in the membrane potential of chloride. This shift has a dramatic effect on neuronal function by changing what would normally be an inhibitory effect into an excitatory one.

Spasticity occurs in a wide range of neuromotor disorders and as a result of neurological trauma ${ }^{3}$. The literature is ripe with various, and sometimes seemingly contradictory, descriptions of the features of spasticity and of the mechanisms leading to them. Despite this apparent confusion, however, there is general agreement that spasticity represents

V. Reggie Edgerton and Roland R. Roy are in the Department of Physiological Science and Brain Research Institute and V. Reggie Edgerton is also in the Department of Neurobiology, University of California, Los Angeles, Los Angeles, California, USA. e-mail: vre@ucla.edu

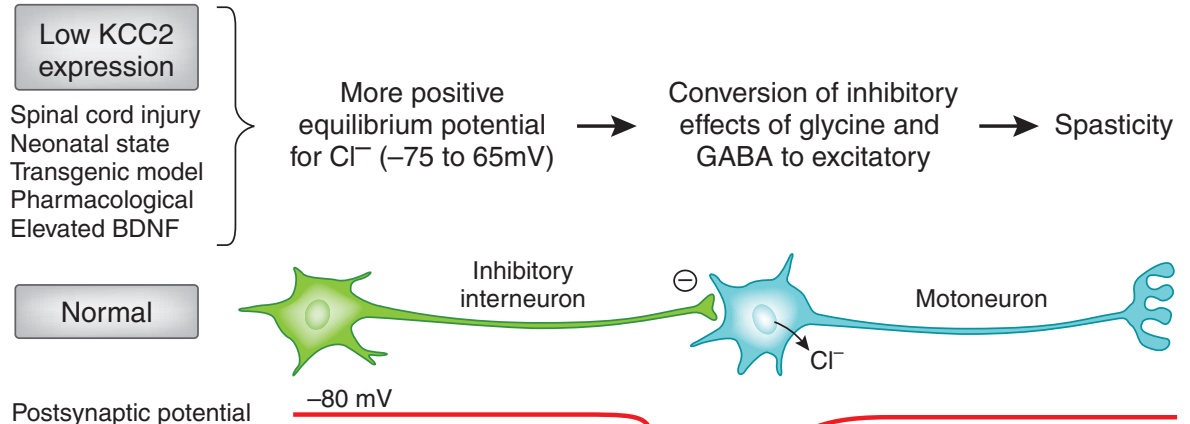

Postsynaptic potential
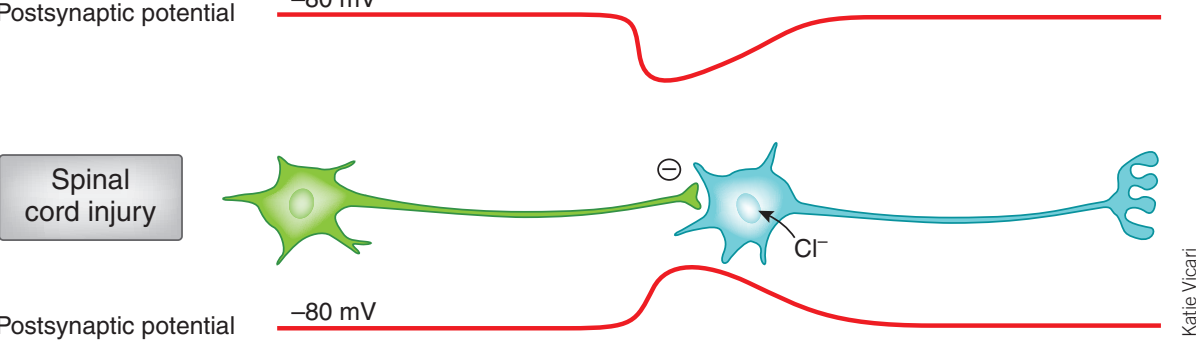

Figure 1 Spasticity results from changes in the regulation of intracellular chloride ions in motoneurons. Expression of KCC2 in motoneuron membranes is reduced after spinal cord injury in neonatal mice, in KCC2-deficient mice, after KCC2 function is pharmacogically blocked, and in response to elevated BDNF. These manipulations of KCC2 expression result in a more positive equilibrium potential for $\mathrm{Cl}^{-}$. This change in the transport property of the membrane results in an alteration in synaptic input that normally would be inhibitory (shown by the downward dip in the postsynaptic potential) to one having a more excitatory effect. This switch contributes to hyperactivity in motoneurons and spasticity. This marked change in the electrophysiological properties of the membrane occurs in the absence of any change in the resting membrane potential $(-80 \mathrm{mV})$.

involuntarily elevated muscular activity and that this activity can be triggered by a variety of sensory inputs, such as cutaneous stimulation $^{4}$.

Although spasticity can originate in muscle, in most cases, elevated muscular activity seems to be linked to more excitable neurons. For example, a number of studies have reported that spastic activity after a spinal cord injury is related to the uncontrolled activation of sodium and calcium persistent inward currents in motoneurons located below the site of the lesion 5 .

Boulenguez et al. ${ }^{2}$ present evidence that another cause of hyperactivity after a spinal cord injury is a lowered expression of $\mathrm{KCC} 2$ in motoneuron membranes. Normally, KCC2 keeps the intracellular concentrations of chloride ions $\left(\mathrm{Cl}^{-}\right)$in neurons low, and the membrane's equilibrium potential is about $-75 \mathrm{mV}$. By inducing spinal cord injury in rats, Boulenguez et al. ${ }^{2}$ show that the equilibrium potential of $\mathrm{Cl}^{-}$in motoneurons below the lesion is about $10 \mathrm{mV}$ more positive than it is before spinal cord injury, leading to increased neuronal activity.

The authors observed similar effects in three other model systems where KCC2 activity is reduced: neonatal mice in which KCC2 is blocked pharmacologically, mice in which the gene for KCC2 is knocked out (KCC2-deficient mice) and mice in which brainderived neurotrophic factor (BDNF) expression 